\title{
3D gel map of Arabidopsis complex I
}

\author{
Katrin Peters, Katharina Belt and Hans-Peter Braun* \\ Institute for Plant Genetics, Faculty of Natural Sciences, Leibniz Universität Hannover, Hannover, Germany
}

\section{Edited by:}

Harvey Millar, The University of Western Australia, Australia

Reviewed by:

Etienne H. Meyer, Max Planck

Society, Germany

Pierre Cardol, Université de Liège,

Belgium

*Correspondence:

Hans-Peter Braun, Institute for Plant

Genetics, Faculty of Natural Sciences,

Leibniz Universität Hannover,

Herrenhäuser Straße 2, 30419

Hannover, Germany

e-mail: braun@genetik.

uni-hannover.de

\begin{abstract}
Complex I has a unique structure in plants and includes extra subunits. Here, we present a novel study to define its protein constituents. Mitochondria were isolated from Arabidopsis thaliana cell cultures, leaves, and roots. Subunits of complex I were resolved by 3D blue-native (BN)/SDS/SDS-PAGE and identified by mass spectrometry. Overall, 55 distinct proteins were found, seven of which occur in pairs of isoforms. We present evidence that Arabidopsis complex I consists of 49 distinct types of subunits, 40 of which represent homologs of bovine complex I. The nine other subunits represent special proteins absent in the animal linage of eukaryotes, most prominently a group of subunits related to bacterial gamma-type carbonic anhydrases. A GelMap http://www. gelmap.de/arabidopsis-3d-complex-i/ is presented for promoting future complex I research in Arabidopsis thaliana.
\end{abstract}

Keywords: mitochondria, OXPHOS system, respiratory chain, NADH dehydrogenase, blue-native, BN/SDS/SDS-PAGE, Arabidopsis thaliana

\section{INTRODUCTION}

The NADH dehydrogenase complex (complex I) of the Oxidative Phosphorylation (OXPHOS) system is present in the cytoplasmic membrane of aerobic bacteria and the inner mitochondrial membrane of eukaryotes. It is composed of two elongated arms: the "membrane arm," and the so-called "peripheral arm" which protrudes into the cytoplasm of the bacterial cell or the matrix of mitochondria (reviewed in Friedrich and Böttcher, 2004; Brandt, 2006; Vogel et al., 2007; Remacle et al., 2008; Zickermann et al., 2008, 2009; Lazarou et al., 2009). The two arms form an L-like structure as originally revealed by electron microscopy (Hofhaus et al., 1991). Very recently, the structure of the entire bacterial enzyme complex has been resolved by Xray crystallography (Baradaran et al., 2013). Complex I represents a NADH:ubiquinone oxidoreductase. Electron transfer entirely takes place within the peripheral arm and involves an electron transfer chain composed of seven FeS clusters (Hinchliffe and Sazanov, 2005). Quinone reduction takes place at the interface between the two arms and was proposed to induce an electrostatical chain reaction throughout the membrane arm which drives proton translocation across the bacterial or mitochondrial membrane (Baradaran et al., 2013).

Complex I is by far the largest complex of the OXPHOS system. In its simplest form, the bacterial complex consists of 14 subunits (seven subunits per arm) and has a molecular mass of about $500 \mathrm{kDa}$. However, in eukaryotes, complex I is much larger and consists of more than 40 subunits. Bovine complex I, which extensively was investigated with respect to its subunit composition, consists of 44 subunits, 16 of which are localized in the peripheral and 28 in the membrane arm (Carroll et al., 2006; Balsa et al., 2012). Complex I composition is remarkably conserved in different eukaryotic lineages (Cardol, 2011). However, some lineage-specific complex I subunits occur (Cardol, 2011).

Additional subunits were especially described for plants. Using electron microscopy, complex I of plants was shown to have a very unique shape (Dudkina et al., 2005; Sunderhaus et al., 2006; Peters et al., 2008; Bultema et al., 2009). It has an extra spherical domain which is attached to the membrane arm at a central position and, like the peripheral arm, protrudes into the mitochondrial matrix. It was shown to include extra subunits which resemble gamma-type carbonic anhydrases (Perales et al., 2005; Sunderhaus et al., 2006). In Arabidopsis, three carbonic anhydrase subunits form part of complex I (termed CA1, CA2, and CA3) and additionally two more derived "carbonic anhydrase-like" proteins (CAL1 and CAL2). Proteomic studies were initiated to systematically characterize complex I subunits in plants (Heazlewood et al., 2003; Cardol et al., 2004; Sunderhaus et al., 2006; Meyer et al., 2008; Klodmann et al., 2010, 2011; Klodmann and Braun, 2011; Li et al., 2013). These projects led to the identification of several proteins homologous to subunits of bovine complex I and some additional subunits specifically occurring in plants. However, resulting protein sets slightly differ between the presented studies (reviewed in Meyer, 2012).

Here, we present a new study to thoroughly characterize complex I subunits in the model plant Arabidopsis thaliana. Our study is based on a 3D gel-electrophoretic approach introduced by Meyer et al. (2008). Using mass spectrometry (MS), 55 complex I proteins were identified, seven of which occur in pairs of isoforms. We present evidence that complex I of Arabidopsis includes at least 49 types of proteins, 40 of which represent homologs of bovine complex I and 9 of which are special to plants. A 3D GelMap is presented at http://www.gelmap.de/ Arabidopsis-3D-complex-I to facilitate future complex I research in Arabidopsis.

\section{MATERIALS AND METHODS PLANT MATERIAL}

A cell culture of Arabidopsis thaliana (Col-0) was established as described by May and Leaver (1993). Callus was maintained 
as suspension culture according to Sunderhaus et al. (2006). Leaves were harvested from 3 weeks old Arabidopsis thaliana (Col0 ) plants grown in soil at long day conditions (16 h light, $8 \mathrm{~h}$ dark) at $22{ }^{\circ} \mathrm{C}$ during the day and $20{ }^{\circ} \mathrm{C}$ at night. Arabidopsis roots were cultured in liquid medium as described by Lee et al. (2011). For this approach, 50-100 seeds of Arabidopsis thaliana Col- 0 were surface-sterilized in $70 \%$ ethanol for $5 \mathrm{~min}$ followed by $5 \mathrm{~min}$ incubation in $5 \%$ bleach/ $0.1 \%$ Tween 20 . Seeds were then washed five times in sterilized water. Length of the single washing steps was increased from $10 \mathrm{~s}$ to finally $5 \mathrm{~min}$. All incubation steps took place in a rotary shaker. After the final washing step an appropriate volume of $0.15 \%$ agarose solution was added to the seeds. The seeds immediately were carefully dispensed on a stainless steel wire mesh platform which is part of the hydroponic culture system adapted from Schlesier et al. (2003). Conditions for hydroponic culture were according to the protocol of Schlesier et al. (2003). Arabidopsis plants were grown under $16 / 8 \mathrm{~h}$ light/dark period with light intensity 100 $125 \mu \mathrm{mol} \mathrm{m}^{-2} \mathrm{~s}^{-1}$ at $22{ }^{\circ} \mathrm{C}$. Liquid medium was replaced with freshly made liquid medium after 2 weeks. After 4 weeks the roots were harvested, pre-washed in root culture medium $[0.38 \%$ (w/v) Gamborg's B5 salt with vitamins, 3\% sucrose, $\mathrm{pH} 5.8$ ] and transferred into Erlenmeyer flasks containing $50 \mathrm{ml}$ root culture medium. The root culture was kept at $22{ }^{\circ} \mathrm{C}$ in the dark under constant agitation at $100 \mathrm{rpm}$ (Lee et al., 2011). It was maintained by transferring small amounts of roots into a new culture flask containing freshly prepared sterilized root culture medium every 3 weeks.

\section{ISOLATION OF MITOCHONDRIA}

Mitochondria from cell culture were isolated as described by Werhahn et al. (2001). Isolation of mitochondria from green leaves and roots was performed according to the protocol of Keech et al. (2005).

\section{D BN/SDS/SDS-PAGE}

One-dimensional blue-native PAGE (1D BN-PAGE) was performed according to Wittig et al. (2006). Mitochondrial membranes were solubilized by digitonin at a concentration of $5 \mathrm{~g} / \mathrm{g}$ mitochondrial protein (Eubel et al., 2003). The two further gel dimensions represented a 2D SDS/SDS-PAGE as originally suggested by Rais et al. (2004). Combining 1D BN-PAGE and 2D SDS/SDS-PAGE was carried out according to Meyer et al. (2008). For this approach, bands corresponding to complex I were excised from the blue-native (BN) gel. Three bands of complex I were used to build a stack on top of a SDS gel (10\% polyacrylamide). Electrophoresis was carried out in the presence of $6 \mathrm{M}$ urea. After end of the electrophoretic run, the lane was cut out from the second gel dimension and incubated in acidic solution (Meyer et al., 2008). The gel strip then was horizontally transferred on top of a third dimension SDS gel (16\% polyacrylamide) and gel electrophoresis was carried out in the absence of urea.

\section{GEL STAINING PROCEDURES}

Polyacrylamide gels were stained with Coomassie Brilliant Blue G250 according to the protocol of Neuhoff et al. (1988, 1990).

\section{PROTEIN IDENTIFICATION BY MASS SPECTROMETRY}

Tryptic digestion of proteins and identification of proteins by MS were performed as described by Klodmann et al. (2010). Procedures were based on peptide separation using the EASYnLC System (Proxeon; Thermo Scientific, Bremen, Germany) and coupled MS analyses using the MicrOTOF-Q II mass spectrometer (Bruker Bremen, Germany). MS data evaluation was carried out using ProteinScape2.1 software (Bruker, Bremen, Germany), the Mascot search engine (Matrix Science, London, UK), and (1) the Arabidopsis protein database ${ }^{1}$ as well as (2) an updated version of the complex I database used by Klodmann et al. (2010). The latter database is also based on the TAIR protein database (release 10) and includes additionally proteins known to co-migrate with complex I on Blue-native gels (like prohibitins). The following Mascot search parameters were used: enzyme, trypsin/P (up to one missed cleavage allowed); global modification, carbamidomethylation $(\mathrm{C})$, variable modifications, acetyl $(\mathrm{N})$, oxidation $(\mathrm{M})$; precursor ion mass tolerance, $15 \mathrm{ppm}$; fragment ion mass tolerance, $0.05 \mathrm{Da}$; peptide charge, $1+, 2+$, and $3+$; instrument type, electrospray ionization quadrupole time of flight. Minimum ion score was 15 , minimum peptide length was four amino acids, significance threshold was set to 0.05 and protein and peptide assessments were carried out if the Mascot Score was greater than 30 for proteins and 20 for peptides.

\section{IMAGE PROCESSING AND DATABASE GENERATION USING GELMAP}

Coomassie-blue stained 3D BN/SDS/SDS gels of complex I were scanned using the Image Scanner III (GE Healthcare). Spot coordinates were generated using Microsoft Office Paint. The gel image and a file containing all relevant MS data including the spot coordinates were exported into the GelMap software package available at www.gelmap.de following the instructions given on the website and in Senkler and Braun (2012).

\section{RESULTS AND DISCUSSION} SEPARATION OF COMPLEX I SUBUNITS BY 3D GEL ELECTROPHORESIS

To further investigate the subunit composition of Arabidopsis complex I, isolated mitochondria from leaves, roots, and cell cultures were analyzed by 3D BN/SDS/SDS-PAGE according to Meyer et al. (2008) (Figure S1 in Supplementary Material). In the first gel dimension intact mitochondrial protein complexes are resolved by BN-PAGE. Bands representing mitochondrial complex I are cut out from the gel and staples of up to three bands are transferred onto the 2D SDS/SDS-PAGE system as published by Rais et al. (2004). The latter electrophoresis system combines the advantages of high resolution SDS-PAGE with differential resolution of hydrophilic versus hydrophobic proteins. The first SDS gel dimension contains $10 \%$ polyacrylamide (PAA) plus $6 \mathrm{M}$ urea while the second SDS gel dimension contains no urea and has a PAA concentration of $16 \%$. On the resulting SDS/SDS gels proteins are dispersed around a diagonal line. This variation in electrophoretic mobility is presumably

\footnotetext{
${ }^{1}$ www.Arabidopsis.org; release TAIR 10
} 
caused by an altered interaction between SDS and proteins in the presence or absence of urea (Rais et al., 2004). Furthermore, highly hydrophobic proteins show a differential electrophoretic mobility in gels with varying PAA concentrations. In low PAA gels, hydrophobic proteins run slightly faster than hydrophilic ones and in high PPA gels the other way round. On the 2D gel system suggested by Rais et al. (2004) hydrophobic proteins run above the diagonal line. Since complex I likewise includes highly hydrophobic and hydrophilic subunits this gel system nicely allows to investigate its composition (Rais et al., 2004; Meyer et al., 2008; Angerer et al., 2011; Dröse et al., 2011). Upon optimization of protocols, 3D BN/SDS/SDS-PAGE of complex I from Arabidopsis cell culture, leaves, and roots allowed to visualize 52 protein spots per fraction based on Coomassie-staining (Figure 1; Figure S2 in Supplementary Material). Variation in subunit composition between the three Arabidopsis tissues was not observed.

\section{ANALYSIS OF COMPLEX I SUBUNITS}

All 52 protein spots of complex I from cell culture and selected subunits of complex I from leaves and roots were analyzed by ESI MS/MS (Figure 2; Table 1; Figure S2 and Tables in Supplementary Material). Overall, 55 distinct proteins were identified. Analyses of two spots in the low-molecular-mass range did not allow identifying any proteins (spots 51 and 52 on Figure 2). Due to spot overlappings, some proteins were detected in more than one spot. The main locations of all proteins (here: highest Mascot score) as well as their secondary locations on the gel are given in Table 1. Overall, 7 out of the 55 subunits of Arabidopsis complex I occur in pairs of isoforms. This reduces the number of distinct types of subunits detected in our complex I fraction to 48. The subunit ND4L was not detected by MS in our or any previous investigation on Arabidopsis complex I which is most likely due to its extreme hydrophobicity (gravy score + 0.976). Systematic analysis of the subunit composition of complex I in the model organism Yarrowia lipolytica also did not led to the identification of this subunit (Abdrakhmanova et al., 2004). ND4L belongs to the "core" set of subunits present in all complex I particles. Its gene is localized on the mitochondrial genome in Arabidopsis, transcribed and edited (Giegé and Brennicke, 1999). We speculate that ND4L is represented by spots 51 or 52 in the $7 \mathrm{kDa}$ range of our 3D gel, both of which could not be identified (Figure 2; Figure S3 in Supplementary Material). ND4L has a calculated mass of $10.9 \mathrm{kDa}$ but is very hydrophobic and therefore should run at $\sim 7 \mathrm{kDa}$ upon SDS-PAGE. We conclude that Arabidopsis complex I consists of at least 49 subunits, 48 of which were detected by our analyses, seven of which occur in pairs of isoforms.

For a limited number of subunits, MS analysis also was carried out for the Arabidopsis leaves and roots fractions (Table 1; Table S1 in Supplementary Material). Identifications confirm the results obtained for the Arabidopsis cell culture. However, in some cases the main locations of corresponding subunits slightly vary between the fractions. It cannot be excluded that these differences are caused by minor gel to gel variations which in some cases made it difficult to precisely assign spots between different fractions. Possible variations in complex I subunit composition between different Arabidopsis fractions should be further addressed by future studies.

Based on previous topological investigations for Arabidopsis and other model organisms (Carroll, 2003; Hunte et al., 2010; Klodmann et al., 2010; Angerer et al., 2011; Cardol, 2011; Dröse et al., 2011), all 49 subunits can be assigned to the membrane or the peripheral arm of complex I. The peripheral arm consists

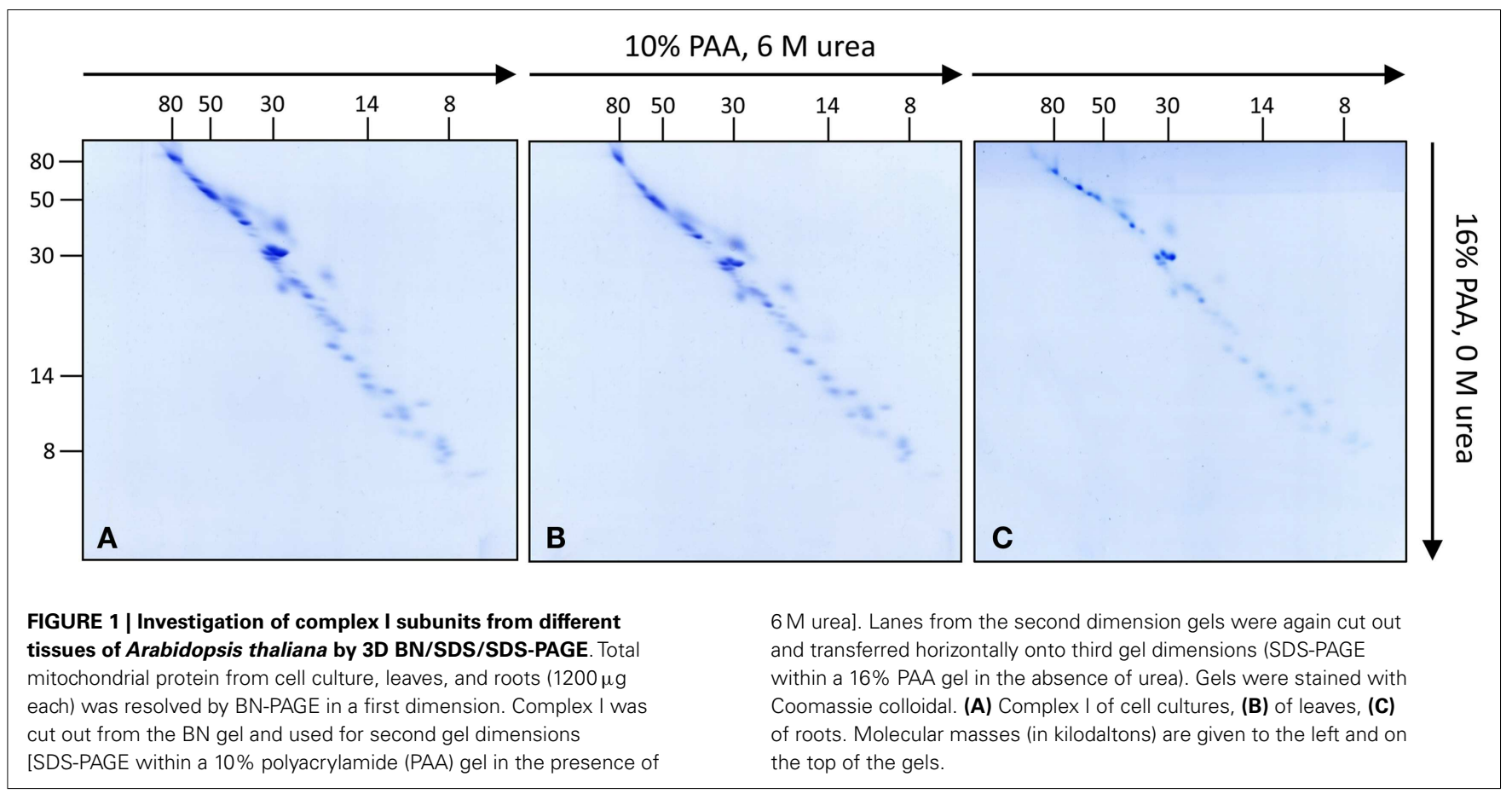



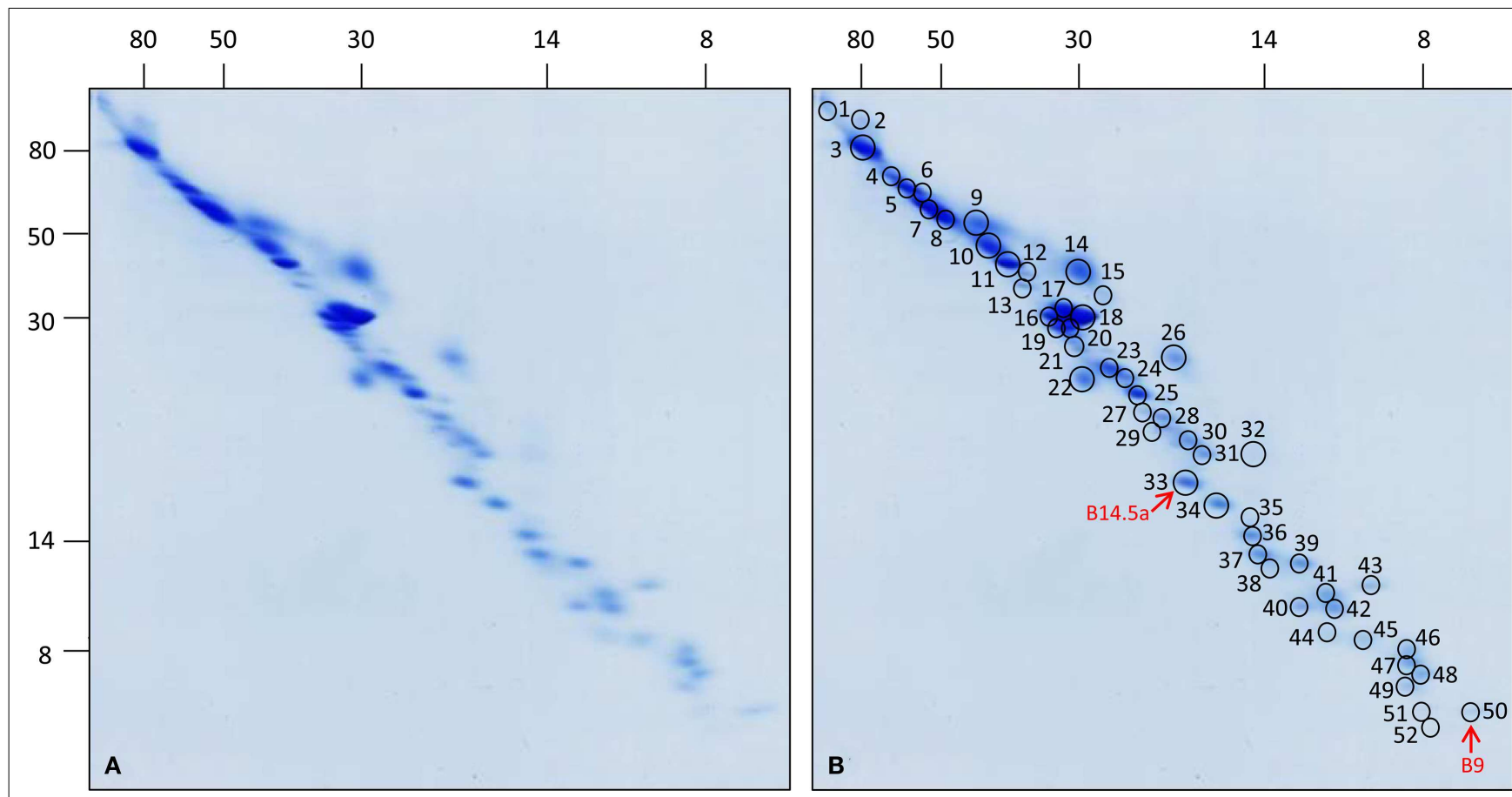

FIGURE 2 | 3D map of complex I from Arabidopsis thaliana cell culture. Total mitochondrial protein ( $1200 \mu \mathrm{g}$ each) was resolved by 3D BN/SDS/SDS-PAGE. (A) Coomassie-stained gel, (B) same gel as in (A) indicating protein spots which have been analyzed by mass spectrometry. Numbers correspond to those given in Table 1. Red arrows indicate the newly identified subunits B14.5a and B9.

Molecular masses (in kilodaltons) are given to the left and on the top of the gels. of 15 subunits, the membrane arm of 34 subunits (Table 1). Five subunits of the membrane arm form part of the so-called carbonic anhydrase (CA/CAL) domain, which is absent in mitochondria of opisthokonts (animals and fungi; Gawryluk and Gray, 2010; Cardol, 2011). Of the 49 subunits, 40 represent homologs of subunits present in bovine complex I (Table 1). Two of these proteins (subunits B14.5a and B9) were identified for the first time in Arabidopsis but previously predicted to form part of complex I by genome analyses (Cardol, 2011). The high number of homologs in bovine and Arabidopsis complex I underlines the remarkable conservation of this protein complex in Eukaryotes (Cardol, 2011). Bovine complex I consists of 44 subunits (Carroll et al., 2006; Balsa et al., 2012), only four of which were not found in Arabidopsis (10 kDa, $42 \mathrm{kDa}$, SDAP, and B17 subunits; Meyer, 2012). On the contrary, Arabidopsis complex I includes nine subunits absent in the bovine complex (for summary, see Figure S4 in Supplementary Material).

Of the nine extra subunits in plants, five represent members of the CA/CAL family. Since deletion of single CA or CAL genes does not cause complete loss of intact complex I (Perales et al., 2005; Sunderhaus et al., 2006; Meyer et al., 2011; Wang et al., 2012a) it cannot be excluded that they present isoforms which alternatively are present in complex I particles. However, deletion of the ca2 gene leads to highly reduced levels of complex I (Perales et al., 2005) indicating that CA2 cannot easily be replaced by CA1 or CA3. Sequence identity between CA1, $\mathrm{CA} 2$, and $\mathrm{CA} 3$ is in the range of $75 \%$. In contrast, sequences of the CAL1 and CAL2 subunits of Arabidopsis are very similar (90\% sequence identity), possibly indicating that these proteins represent isoforms. Indeed, deletion of the call or cal2 gene in Arabidopsis does not visibly affect Arabidopsis development but the double mutant is not viable (Wang et al., 2012a). Considering the size of the CA/CAL domain upon single particle EM of Arabidopsis complex I it was concluded that it consists of at least three copies of CA/CAL proteins (Sunderhaus et al., 2006). Further experiments have to be carried out in order to clarify the number of CA/CAL subunits per individual complex I particles.

The plant-specific GLDH subunit binds to three complex I assembly intermediates of 420,480 , and $850 \mathrm{kDa}$ (Schertl et al., 2012) but so far was not detected in preparations of intact complex I. Our data point to the possibility that GLDH also binds to the intact complex. However, it cannot be excluded that the $1000 \mathrm{kDa}$ complex I band excised from the BN gel also included small amounts of the band representing the $850 \mathrm{kDa}$ subcomplex. Three further plant-specific subunits were detected on our 3D gels: P1, P2, and a protein encoded by At1g18320. The P1 and $\mathrm{P} 2$ proteins were consistently detected in complex I fractions from plants (Meyer, 2012). Both form part of the membrane arm (Sunderhaus et al., 2006). The At1g18320 protein was previously found to co-migrate with complex I on a BN/SDS gel (Klodmann et al., 2011). However, its status representing an integral complex I subunit in Arabidopsis should be further investigated. 
Table 1 | Complex I subunits in Arabidopsis thaliana.

\begin{tabular}{|c|c|c|c|c|c|}
\hline \multicolumn{2}{|c|}{ Subunit $^{1}$} & \multirow[t]{2}{*}{ Accession $^{2}$} & \multicolumn{2}{|c|}{ Spot $^{3}$} & \multirow[t]{2}{*}{ Organ $^{4}$} \\
\hline Plant subunit & Bovine homolog & & Main spot & Further spots & \\
\hline \multicolumn{6}{|l|}{ Membrane arm } \\
\hline 15 kDa-1 & $15 \mathrm{kDa}$ & At3g62790 & 41 & & c \\
\hline $15 \mathrm{kDa}-2$ & $15 \mathrm{kDa}$ & At2g47690 & 41 & & c \\
\hline AGGG & AGGG & At1g76200 & 47 & & c \\
\hline ASHI & ASHI & At5g47570 & 44 & & c \\
\hline B9 & B9 & At2g46540 & 50 & & c \\
\hline B12-1 & B12 & At1g14450 & 45 & & $c, 1$ \\
\hline B12-2 & B12 & At2g02510 & 45 & & $c, 1$ \\
\hline B14 & B14 & At3g12260 & 36 & & $\mathrm{c}$ \\
\hline B14.5b & B14.5b & At4g20150 & 47 & & $\mathrm{c}, \mathrm{l}$ \\
\hline B14.7 & B14.7 & At2g42210 & 33 & & $c, 1$ \\
\hline B15 & B15 & At2g31490 & 46 & & $c, 1$ \\
\hline B16.6-1 & B16.6 & At1g04630 & 34 & & $c, l$ \\
\hline B16.6-2 & B16.6 & At2g33220 & 34 & & c \\
\hline B18 & B18 & At2g02050 & 38 & 37 & $c, l, r$ \\
\hline B22 & B22 & At4g34700 & 35 & & c \\
\hline ESSS-1 & ESSS & At2g42310 & 42 & & c \\
\hline ESSS-2 & ESSS & At3g57785 & 42 & & c \\
\hline KFYI & KFYI & At4g00585 & 41 & 32 & c \\
\hline MNLL & MNLL & At4g16450 & 40 & & $\mathrm{c}, \mathrm{l}, \mathrm{r}$ \\
\hline MWFE & MWFE & At3g08610 & 49 & & $c, 1$ \\
\hline ND1 & ND1 & AtMg00516/AtMg01120/AtMg012755 & 26 & & $c, 1$ \\
\hline ND2 & ND2 & AtMg00285/AtMg013205 & 14 & & $c, l, r$ \\
\hline ND3 & ND3 & AtMg00990 & 43 & & c \\
\hline ND4 & ND4 & AtMg00580 & 14 & & c \\
\hline ND4L & ND4L & AtMg00650 & - & & - \\
\hline ND5 & ND5 & AtMg00060/AtMg00513/AtMg006655 & 9 & 2 & $c, 1$ \\
\hline ND6 & ND6 & AtMg00270 & 15 & & $\mathrm{c}$ \\
\hline PDSW-2 & PDSW & At1g49140 & 36 & & $c, l, r$ \\
\hline PDSW-1 & PDSW & At3g18410 & 36 & & $c, l, r$ \\
\hline PGIV-1 & PGIV & At3g06310 & 37 & & $\mathrm{c}$ \\
\hline PGIV-2 & PGIV & At5g18800 & 38 & 37 & c \\
\hline GLDH & - & At3g47930 & 4 & & c \\
\hline P1 & - & At1g67350 & 39 & & $c, 1$ \\
\hline P2 & - & At2g27730 & 37 & & $c, l, r$ \\
\hline At1g18320 & - & At1g18320 & 29 & & $\mathrm{C}$ \\
\hline \multicolumn{6}{|c|}{ Carbonic anhydrase domain (membrane arm) } \\
\hline CA1 & - & At1g19580 & 16 & $17,18,19,20$ & $c, 1$ \\
\hline CA2 & - & At1g47260 & 16 & $13,17,18,19,20$ & $c, 1$ \\
\hline CA3 & - & At5g66510 & 21 & 19,20 & $c, 1$ \\
\hline CAL1 & - & At5g63510 & 23 & & $c, 1$ \\
\hline CAL2 & - & At3g48680 & 23 & 22,24 & $c, l, r$ \\
\hline \multicolumn{6}{|l|}{ Peripheral arm } \\
\hline $13 \mathrm{kDa}$ & $13 \mathrm{kDa}$ & At3g03070 & 44 & & c \\
\hline $18 \mathrm{kDa}$ & $18 \mathrm{kDa}$ & At5g67590 & 33 & & $c, 1$ \\
\hline $24 \mathrm{kDa}$ & $24 \mathrm{kDa}$ & At4g02580 & 21 & & $c, l, r$ \\
\hline $39 \mathrm{kDa}$ & $39 \mathrm{kDa}$ & At2g20360 & 11 & 12 & $c, l, r$ \\
\hline $51 \mathrm{kDa}$ & $51 \mathrm{kDa}$ & At5g08530 & 8 & $5,6,7,9$ & $c, 1$ \\
\hline $75 \mathrm{kDa}$ & $75 \mathrm{kDa}$ & At5g37510 & 3 & $1,2,4$ & $c, 1$ \\
\hline B8 & B8 & At5g47890 & 42 & 41 & $c, l$ \\
\hline B13 & B13 & At5g52840 & 28 & 27,29 & $c, l, r$ \\
\hline B14.5a & B14.5a & At5g08060 & 33 & & c \\
\hline B17.2 & B17.2 & At3g03100 & 31 & & c \\
\hline ND7 & 49 kDa & AtMg00510 & 10 & & $c, 1$ \\
\hline ND9 & $30 \mathrm{kDa}$ & AtMg00070 & 25 & & $c, I, r$ \\
\hline PSST & PSST & At5g11770 & 30 & & $c, l, r$ \\
\hline SGDH & SGDH & At1g67785 & 48 & & $c, 1$ \\
\hline TYKY-1 & TYKY & At1g79010 & 22 & & $\mathrm{c}, \mathrm{l}$ \\
\hline TYKY-2 & TYKY & At1g16700 & 22 & & $c, 1$ \\
\hline
\end{tabular}




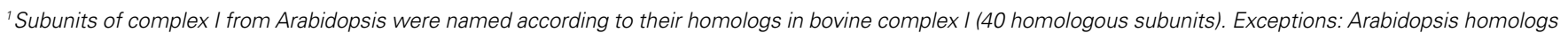

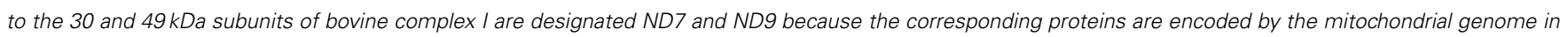

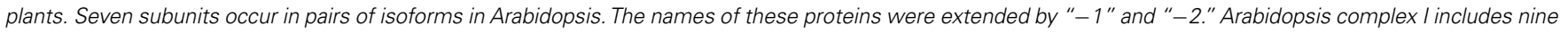

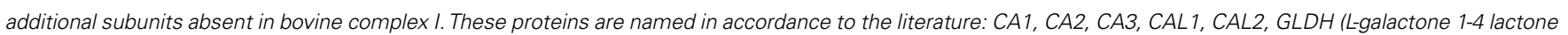
dehydrogenase), P1, P2, and At1g18320.

${ }^{2}$ Accession numbers as given by TAIR http:// www.arabidopsis.org.

${ }^{3}$ Spot number in accordance with Figure 2.

${ }^{4}$ Organ/culture in which the subunit was identified; c, cell culture; I, leaf; r, root.

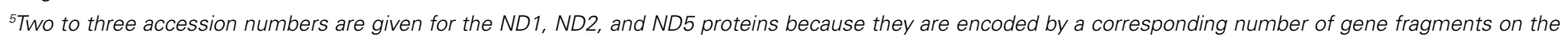

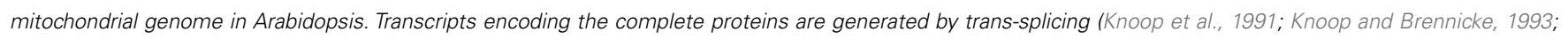
Lippok et al., 1996).

Table 2 | Candidates of additional complex I subunits in Arabidopsis thaliana.

\begin{tabular}{lll}
\hline Accession & Evidence & Remark \\
\hline At5g14105 & Klodmann et al. (2010), Klodmann and & Subunit P3 ${ }^{1}$ \\
& Braun (2011) & \\
At1g68680 & Meyer et al. (2008) & Similar to TIM22 \\
At3g10110 & Klodmann et al. (2011) & \\
At1g72170 & Klodmann et al. (2011) & \\
At2g28430 & Klodmann et al. (2011) & Similar to TIM23 \\
At1g72750 & Wang et al. (2012b) &
\end{tabular}

${ }^{1}$ At5g14105 was suggested to be named P3 in Meyer (2012).

\section{FURTHER COMPLEX I SUBUNITS IN PLANTS?}

In previous investigations based on BN-PAGE six additional complex I proteins were identified in Arabidopsis (summarized in Meyer, 2012): At5g14105 (Klodmann et al., 2010; Klodmann and Braun, 2011), At1g68680 (Meyer et al., 2008), At1g72170, At3g10110 and At2g28430 (Klodmann et al., 2011), and At1g72750 (Wang et al., 2012b) (Table 2). However, detection of these proteins is not consistent. It currently cannot be excluded that these proteins co-migrate with complex I on blue-native gels but form part of separate complexes. Interestingly, some of these proteins are known components of the pre-protein translocase of the inner mitochondrial membrane, the TIM complex (At1g72750 and At3g10110; the latter protein represents an isoform of At1g18320 which was identified in the course of our current study; Table 1). It recently has been suggested that complex I and the TIM complex are physically linked in plant mitochondria (Murcha et al., 2012).

\section{D REFERENCE MAP OF COMPLEX I}

To facilitate identifying complex I subunits upon 3D BN/SDS/SDS-PAGE, a GelMap was generated for the MS dataset of the gel presented in Figure 2. GelMap is a software tool for the building and presentation of proteome reference maps (www.gelmap.de; Senkler and Braun, 2012). In contrast to alternative software packages, it allows assignment of multiple proteins per protein spot and at the same time functional annotation of all proteins. By clicking onto protein spots, widespread information is offered. Several GelMaps on Arabidopsis mitochondria are presented at the GelMap homepage, including a map on SDS-induced complex I subcomplexes ${ }^{2}$.

For the 3D GelMap of Arabidopsis complex I, the 55 identified proteins are grouped into functional categories according to their localization within the peripheral arm, the membrane arm, or the carbonic anhydrase domain attached to the membrane arm (Figure 3; http://www.gelmap.de/arabidopsis-3d-complex-i/). Furthermore, the six candidates for additional complex I subunits are given in another category. The proteins of the latter category are linked to an "extra" spot below the gel. By clicking onto any protein spot on the map, all included proteins are displayed. Proteins are sorted according to their MASCOT scores. Upon clicking onto an individual protein, a tooltip opens which includes additional information. Extensive further information on each protein is offered by links to several external databases. The new GelMap is intended to be a helpful tool for future complex I research in Arabidopsis.

\section{ACKNOWLEDGMENTS}

Katrin Peters was supported by the "Wege in die Forschung II" program offered by Leibniz University Hannover. We acknowledge support by Deutsche Forschungsgemeinschaft (DFG) and Open Access Publishing Fund of Leibniz Universität Hannover.

\section{SUPPLEMENTARY MATERIAL}

The Supplementary Material for this article can be found online at: http://www.frontiersin.org/Plant_Proteomics/10.3389/ fpls.2013.00153/abstract

\section{Figure S1 | Principle of 3D BN/SDS/SDS-PAGE.}

Figure S2 | Replicates of 3D BN/SDS/SDS gels for complex I from cell cultures of Arabidopsis.

Figure S3 | Regions on 3D BN/SDS/SDS gels showing the smallest complex I subunits. Gels were Coomassie stained (left, middle) or silver stained (right). The three smallest proteins (corresponding to spots 50, 51, and 52 on Figure 2) only become clearly visible upon silver staining. Spot 50 represents the B9 subunit. Spot 51 might represent subunit ND4L. Spot 52 could not be identified.

Figure S4 | Species specific complex I subunits in B. taurus and A. thaliana.

${ }^{2}$ http://www.gelmap.de/177 


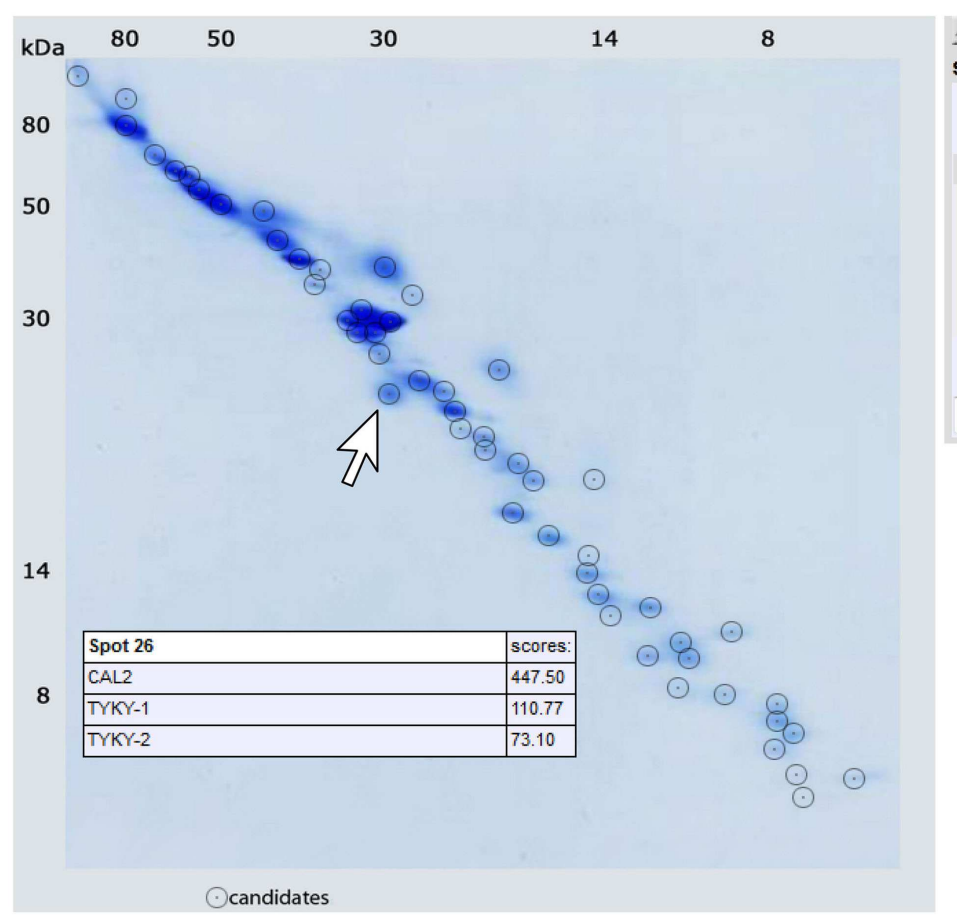

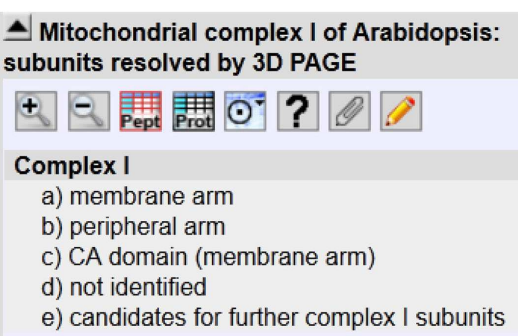

Search (protein name, accession number):
FIGURE 3 | GelMap of complex I as resolved by 3D BN/SDS/SDSPAGE (http://www.gelmap.de/arabidopsis-3d-complex-i/). Upon hovering with the cursor over a spot, a tooltip including information on all included proteins is opened. In the example given on the figure, the indicated spot includes the CAL2 protein and two isoforms of the TYKY subunit. Upon clicking into the spot the protein names are converted into stable links which can be used to obtain further information. Protein information also can be obtained by clicking into the menu given to the right or by entering protein names or accessions into the search field below the menu.
Figure S5 | Identity of complex I subunits of Arabidopsis upon analysis by 3D BN/SDS/SDS PAGE.

\section{Table S1 | Protein table of the GelMap}

(http://www.gelmap.de/arabidopsis-3d-complex-i/).

\section{REFERENCES}

Abdrakhmanova, A., Zickermann, V., Bostina, M., Radermacher, M., Schägger, H., Kerscher, S., et al. (2004). Subunit composition of mitochondrial complex I from the yeast Yarrowia lipolytica. Biochim. Biophys. Acta 1658, 148-156. doi:10.1016/j.bbabio.2004. 04.019

Angerer, H., Zwicker, K., Wumaier, Z., Sokolova, L., and Heide, H. (2011). A scaffold of accessory subunits links the peripheral arm and the distal proton-pumping module of mitochondrial complex I. Biochem. J. 437, 279-288. doi:10.1042/BJ20110359

Balsa, E., Marco, R., Perales-Clemente, E., Szklarczyk, R., Calvo, E., Landazuri, M. O., et al. (2012). NDUFA4 is a subunit of complex IV of the mammalian electron transport chain. Cell Metab. 16, 378-386. doi:10.1016/j.cmet.2012.07.015

Baradaran, R., Berrisford, J. M., Minhas, G. S., and Sazanov, L. A. (2013).
Crystal structure of the entire respiratory complex I. Nature 494, 443-448. doi:10.1038/nature11871

Brandt, U. (2006). Energy converting NADH:quinone oxidoreductase (complex I). Annu. Rev. Biochem. 75, 69-92. doi:10.1146 /annurev.biochem.75.103004.142539 Bultema, J., Braun, H.-P., Boekema, E., and Kouril, R. (2009). Megacomplex organization of the oxidative phosphorylation system by structural analysis of respiratory supercomplexes from potato. Biochim. Biophys. Acta 1787, 60-67. doi:10.1016/j.bbabio.2008.10.010

Cardol, P. (2011). Mitochondrial NADH:ubiquinone oxidoreductase (complex I) in eukaryotes: a highly conserved subunit composition highlighted by mining of protein databases. Biochim. Biophys. Acta 1807, 1390-1397. doi:10.1016/j.bbabio.2011.06.015

Cardol, P., Vanrobaeys, F., Devreese, B., van Beeumen, J., Matagne,

Table S2 | Protein table of complex I subunits in leaves of Arabidopsis thaliana.

Table S3 | Protein table of complex I subunits in roots of Arabidopsis thaliana.

R. F., and Remacle, C. (2004). Higher plant-like subunit composition of mitochondrial complex I from Chlamydomonas reinhardtii: 31 conserved components among eukaryotes. Biochim. Biophys. Acta 1658, 212-224. doi:10.1016/j.bbabio.2004.06.001

Carroll, J. (2003). Analysis of the subunit composition of complex I from bovine heart mitochondria. Mol. Cell. Proteomics 2, 117-126.

Carroll, J., Fearnley, I. M., Skehel, J. M., Shannon, R. J., Hirst, J., and Walker, J. E. (2006). Bovine complex I is a complex of 45 different subunits. J. Biol. Chem. 281, 32724-32727. doi:10.1074/jbc.M60713 5200

Dröse, S., Krack, S., Sokolova, L., Zwicker, K., Barth, H. D., Morgner, N., et al. (2011). Functional dissection of the proton pumping modules of mitochondrial complex I. PLoS Biol. 9:e1001128. doi:10.1371/journal.pbio.1001128
Dudkina, N. V., Eubel, H., Keegstra, W., Boekema, E. J., and Braun, H.-P. (2005). Structure of a mitochondrial supercomplex formed by respiratory-chain complexes I and III. Proc. Natl. Acad. Sci. U.S.A. 102, 3225-3229. doi:10.1073/pnas.040887 0102

Eubel, H., Jänsch, L., and Braun, H.-P. (2003). New insights into the respiratory chain of plant mitochondria. Supercomplexes and a unique composition of complex II. Plant Physiol. 133, 274-286. doi:10.1104/pp.103. 024620

Friedrich, T., and Böttcher, B. (2004). The gross structure of the respiratory complex I: a Lego System. Biochim. Biophys. Acta 1608, 1-9. doi:10.1016/j.bbabio.2003. 10.002

Gawryluk, R. M., and Gray, M. W. (2010). Evidence for an early evolutionary emergence of gamma-type carbonic anhydrase as components of mitochondrial 
respiratory complex I. BMC Evol. Biol. 10:176. doi:10.1186/1471-2148-10-176

Giegé, P., and Brennicke, A. (1999). RNA editing in Arabidopsis mitochondria effects $441 \mathrm{C}$ to $\mathrm{U}$ changes in ORFs. Proc. Natl. Acad. Sci. U.S.A. 96, 15324-15329. doi:10.1073/pnas.96.26.15324

Heazlewood, J. L., Howell, K. A., and Millar, A. H. (2003). Mitochondrial complex I from Arabidopsis and rice: orthologs of mammalian and fungal components coupled with plant-specific subunits. Biochim. Biophys. Acta 1604, 159-169. doi:10.1016/S00052728(03)00045-8

Hinchliffe, P., and Sazanov, L. A. (2005). Organization of ironsulfur clusters in respiratory complex I. Science 309, 771-774. doi:10.1126/science.1113988

Hofhaus, G., Weiss, H., and Leonard, K. (1991). Electron microscopic analysis of the peripheral and membrane parts of mitochondrial NADH dehydrogenase (complex I). J. Mol. Biol. 221, 1027-1043. doi:10.1016/00222836(91)80190-6

Hunte, C., Zickermann, V., and Brandt, U. (2010). Functional modules and structural basis of conformational coupling in mitochondrial complex I. Science 329, 448-451. doi:10.1126/science. 1191046

Keech, O., Dizengremel, P., and Gardeström, P. (2005). Preparation of leaf mitochondria from Arabidopsis thaliana. Physiol. Plant 124, 403-409. doi:10.1111/j.13993054.2005.00521.x

Klodmann, J., and Braun, H.-P. (2011). Proteomic approach to characterize mitochondrial complex I from plants. Phytochemistry 72, 1071-1080. doi:10. 1016/j.phytochem.2010.11.012

Klodmann, J., Senkler, M., Rode, C., and Braun, H.-P. (2011). Defining the protein complex proteome of plant mitochondria. Plant Physiol. 157, 587-598. doi:10.1104/pp.111.182352

Klodmann, J., Sunderhaus, S., Nimtz, M., Jansch, L., and Braun, H.-P. (2010). Internal architecture of mitochondrial complex I from Arabidopsis thaliana. Plant Cell 22, 797-810. doi:10.1105/tpc.109.073726

Knoop, V., and Brennicke, A. (1993). "Group II introns in plant mitochondria - trans-splicing, RNA editing, evolution and promiscuity," in Plant Mitochondria, eds A. Brennicke and U. Kück
(Weinheim: VCH Verlagsgesellschaft), 221-232.

Knoop, V., Schuster, W., Wissinger, B., and Brennicke, A. (1991). Trans splicing integrates an exon of 22 nucleotides into the nad5 mRNA in higher plant mitochondria. EMBO J. 10, 3483-3493.

Lazarou, M., Thorburn, D. R., Ryan, M. T., and McKenzie, M. (2009). Assembly of mitochondrial complex I and defects in disease. Biochim. Biophys. Acta 1793, 78-88. doi:10.1016/j.bbamcr.2008. 04.015

Lee, C. P., Eubel, H., O’Toole, N., and Millar, A. H. (2011). Combining proteomics of root and shoot mitochondria and transcript analysis to define constitutive and variable components in plant mitochondria. Phytochemistry 72, 1092-1108. doi:10.1016/j.phytochem. 2010.12.004

Li, L., Nelson, C. J., Carrie, C., Gawryluk, R. M., Solheim, C., Gray, M. W., et al. (2013). Subcomplexes of ancestral respiratory complex I subunits rapidly turn over in vivo as productive assembly intermediates in Arabidopsis. J. Biol. Chem. 288, 5707-5717. doi:10.1074/jbc.M112. 432070

Lippok, B., Brennicke, A., and Unseld, M. (1996). The rps4-gene is encoded upstream of the nad2gene in Arabidopsis mitochondria. Biol. Chem. Hoppe-Seyler 377, 251-257. doi:10.1515/bchm3.1996. 377.4.251

May, M. J., and Leaver, C. J. (1993). Oxidative stimulation of glutathione synthesis in Arabidopsis thaliana suspension cultures. Plant Physiol. 103, 621-627.

Meyer, E. H. (2012). Proteomic investigations of complex I composition: how to define a subunit? Front. Plant Sci. 3:106. doi:10.3389/fpls.2012.00106

Meyer, E. H., Solheim, C., Tanz, S. K., Bonnard, G., and Millar, A. H. (2011). Insights into the composition and assembly of the membrane arm of plant complex I through analysis of subcomplexes in Arabidopsis mutant lines. J. Biol. Chem. 286, 26081-26092. doi:10.1074/jbc.M110. 209601

Meyer, E. H., Taylor, N. L., and Millar, A. H. (2008). Resolving and identifying protein components of plant mitochondrial respiratory complexes using three dimensions of gel electrophoresis. J. Proteome Res. 7, 786-794. doi:10.1021/ pr700595p
Murcha, M. W., Wang, Y., and Whelan, J. (2012). A molecular link between mitochondrial preprotein transporters and respiratory chain complexes. Plant Signal. Behav. 7, 1594-1597. doi:10.4161/psb. 22250

Neuhoff, V., Stamm, R., and Eibl, H. (1988). Clear background and highly sensitive protein staining with coomassie blue dyes in polyacrylamide gels: a systematic analysis. Electrophoresis 11, 427-448.

Neuhoff, V., Stamm, R., Pardowitz, I., Arold, N., Ehrhardt, W., and Taube, D. (1990). Essential problems in quantification of proteins following colloidal staining with Coomassie brilliant blue dyes in polyacrylamide gels, and their solution. Electrophoresis 11, 101-117. doi:10.1002/elps.115011 0202

Perales, M., Eubel, H., Heinemeyer, J., Colaneri, A., Zabaleta, E., and Braun, H.-P. (2005). Disruption of a nuclear gene encoding a mitochondrial gamma carbonic anhydrase reduces complex I and supercomplex I+III2 levels and alters mitochondrial physiology in Arabidopsis. J. Mol. Biol. 350 263-277. doi:10.1016/j.jmb.2005. 04.062

Peters, K., Dudkina, N. V., Jänsch, L., Braun, H.-P., and Boekema E. J. (2008). A structural investigation of complex I and I+III2 supercomplex from Zea mays at 11-13 A resolution: assignment of the carbonic anhydrase domain and evidence for structural heterogeneity within complex I. Biochim. Biophys. Acta 1777, 84-93. doi:10.1016/j.bbabio.2007. 10.012

Rais, I., Karas, M., and Schägger, H. (2004). Two-dimensional electrophoresis for the isolation of integral membrane proteins and mass spectrometric identification. Proteomics 4, 2567-2571. doi:10.1002/pmic.200400829

Remacle, C., Barbieri, M. R., Cardol, P., and Hamel, P. P. (2008). Eukaryotic complex I: functional diversity and experimental systems to unravel the assembly process. Mol. Genet. Genomics 280, 93-110. doi:10.1007/s00438-008-0350-5

Schertl, P., Sunderhaus, S., Klodmann, J., Grozeff, G. E. G., Bartoli, C. G., and Braun, H.-P. (2012). L-galactono1,4-lactone dehydrogenase (GLDH) forms part of three subcomplexes of mitochondrial complex I in Arabidopsis thaliana. J. Biol. Chem. 287,
14412-14419. doi:10.1074/jbc.M111. 305144

Schlesier, B., Bréton, F., and Mock, H.-P. (2003). A hydroponic culture system for growing Arabidopsis thaliana plantlets under sterile conditions. Plant Mol. Biol. Rep. 21, 449-456. doi:10.1007/ BF02772594

Senkler, M., and Braun, H.-P. (2012). Functional annotation of 2D protein maps: the GelMap portal. Front. Plant Sci. 3:87. doi:10.3389/fpls.2012.00087

Sunderhaus, S., Dudkina, N., Jansch, L., Klodmann, J., Heinemeyer, J., Perales, M., et al. (2006). Carbonic anhydrase subunits form a matrix-exposed domain attached to the membrane arm of mitochondrial complex I in plants. J. Biol. Chem. 281, 6482-6488. doi:10.1074/jbc.M51154 2200

Vogel, R. O., Smeitink, J. A. M., and Nijtmans, L. G. J. (2007). Human mitochondrial complex I assembly: a dynamic and versatile process. Biochim. Biophys. Acta 1767, 1215-1227. doi:10.1016/j.bbabio. 2007.07.008

Wang, Q., Fristedt, R., Yu, X., Chen, Z., Liu, H., Lee, Y., et al. (2012a). The gamma-carbonic anhydrase subcomplex of mitochondrial complex I is essential for development and important for photomorphogenesis of Arabidopsis. Plant Physiol. 160, 1373-1383. doi:10.1104/pp.112. 204339

Wang, Y., Carrie, C., Giraud, E., Elhafez, D., Narsai, R., Duncan, O., et al. (2012b). Dual location of the mitochondrial preprotein transporters B14.7 and Tim23-2 in complex I and the TIM17:23 complex in Arabidopsis links mitochondrial activity and biogenesis. Plant Cell 24, 2675-2695. doi:10.1105/tpc.112. 098731

Werhahn, W., Niemeyer, A., Jansch, L., Kruft, V., Schmitz, U. K., and Braun, H. (2001). Purification and characterization of the preprotein translocase of the outer mitochondrial membrane from Arabidopsis. Identification of multiple forms of TOM20. Plant Physiol. 125, 943-954. doi:10.1104/pp.125. 2.943

Wittig, I., Braun, H.-P., and Schägger, H. (2006). Blue native PAGE. Nat. Protoc. 1, 418-428. doi:10.1038/nprot.2006.62

Zickermann, V., Drose, S., Tocilescu, M. A., Zwicker, K., Kerscher, S., and Brandt, U. (2008). Challenges in elucidating structure and 
mechanism of proton pumping NADH:ubiquinone oxidoreductase (complex I). J. Bioenerg. Biomembr. 40, 475-483. doi:10.1007/s10863008-9171-9

Zickermann, V., Kerscher, S., Zwicker, K., Tocilescu, M. A., Radermacher, M., and Brandt, U. (2009). Architecture of complex $I$ and its implications for electron transfer and proton pumping. Biochim. Biophys. Acta 1787, 574-583. doi:10.1016/j.bbabio.2009.01.012

Conflict of Interest Statement: The authors declare that the research was conducted in the absence of any commercial or financial relationships that could be construed as a potential conflict of interest.
Received: 22 March 2013; accepted: 04 May 2013; published online: 04 June 2013.

Citation: Peters $K$, Belt $K$ and Braun H-P (2013) 3D gel map of Arabidopsis complex I. Front. Plant Sci. 4:153. doi: 10.3389/fpls.2013.00153

This article was submitted to Frontiers in Plant Proteomics, a specialty of Frontiers in Plant Science.
Copyright $(92013$ Peters, Belt and Braun. This is an open-access article distributed under the terms of the Creative Commons Attribution License, which permits use, distribution and reproduction in other forums, provided the original authors and source are credited and subject to any copyright notices concerning any third-party graphics etc. 